\title{
Characterization of Microbial Community Composition in the Fermentation Starter (Daqu) Reveals a Distinct Dominant Species Combination Potentially Contributing to the Flavor of Chinese Gujingtribute Liquor
}

\author{
Hui-min ZHANG ${ }^{1}$, Hong-kui HE ${ }^{2, a, *}$, Run-jie $\mathrm{CAO}^{2}$, Lei LIU ${ }^{1}$, Zhi-hui \\ TANG $^{2}$, An-jun $\mathrm{LI}^{2}$ and Zhi-zhou ZHANG ${ }^{1,3, \mathrm{~b},{ }^{*}}$ \\ ${ }^{1}$ School of Chemistry and Chemical Engineering, Marine Anti-fouling Engineering \\ Technology Center of Shandong Province, Harbin Institute of Technology, Harbin, \\ China 150006 \\ ${ }^{2}$ The Anhui GuJingTribute Liquor Ltd, Bozhou, Anhui, China 236800 \\ ${ }^{3}$ Shandong Gredmedic Co., Ltd., Weihai, China 264209 \\ ${ }^{*}$ Corresponding authors \\ ahongkui896@163.com \\ bzhangzzbiox@hitwh.edu.cn
}

Keywords: Fermentation, Daqu, Pantoea agglomerans, Thermomyces lanuginosus, Thermoascus aurantiacus.

\begin{abstract}
In this study the microbial composition in the starter (Daqu) of Chinese Gujing Tribute liquor was determined by employing near full length ribosome gene library sequencing approach. Gujing Tribute Daqu has a distinct microbial community structure by comparison with several other important types of Chinese liquor; especially The rmomyces only occurred in Gujing Tribute Daqu, not in any other types of Daqu as most dominant species. Besides, there are five distinct species as most dominant ones in Gujing Tribute Daqu: Pantoea agglomerans, Pantoea vagans, Talaromyces thermophiles, Thermomyces lanuginosus, and Thermoascus aurantiacus, the latter two species both capable of producing a series of useful enzymes for fermentation purposes and some flavor precursor synthesis. Though the distinct dominating species are mainly the ones degrading the feedstock, their potential roles in the flavor determination of Gujing Tribute liquor are very valuable for further study.
\end{abstract}

\section{Introduction}

The production of most Chinese liquors starts from Daqu, the primary microbial source for liquor fermentation [1]. Daqu is the fermentation starter with an aggregation of microorganisms and abundant saccharifying enzymes originating from these microorganisms. The fermentation starter is associated with the degradation of different kinds of starch (from wheat, rice, sorghum, maize, glutinous rice, etc) and the production of alcohol, during which liquor aroma substances are also synthesized. The difference among different types of liquor originates first from Daqu's composition of active micro biota and one to several types of cereals. The combination of cereal types, air, water quality, production process, sanitary conditions, additives, and other geographical parameters (temperature, moisture etc.) altogether determines the nature of Daqu. Basically all types of traditionally-produced Chinese Daqu and their properties 
can't be well replicated in other areas. Knowledge of the functional correlation between Daqu microbiota and liquor aroma profile is still very poor.

GujingTribute liquor has a history of over 1800 years in China with distinct flavor and metabolite profiles [2-5]. The Daqu for GujingTribute liquor production is a mixture with a specific ratio between high-temperature Daqu (H-Daqu) and medium high-temperature Daqu (MH-Daqu), which contributes to the unique flavor of GujingTribute liquor as a strong-flavor type. In our knowledge, no reports have described the compositional comparison of those most dominant species between GujingTribute and other major types of Chinese liquor for their Daqus in the context of the relationship between Daqu microbiota and liquor flavor. In this study, the authors characterized microbial compositions of two different types of GujingTribute Daqu, and compared the most dominant species with those of other representative brands of Chinese liquor. Considering that near full-length 16S rRNA genes sequences are still best for accurate estimations and better classifications of microbial populations, we employed ribosome DNA library sequencing approach to clone and Sanger-sequence near-full-length ribosome genes in the selected GujingTribute Daqu samples.

\section{Materials and methods}

\section{Fermentation Samples}

Randomly selected twelve H-Daqu bricks were smashed and well-mixed into one $\mathrm{H}$-Daqu sample, and randomly selected twelve MH-Daqu bricks were smashed and well-mixed into one MH-Daqu sample. Samples were immediately stored at $-80^{\circ} \mathrm{C}$ in Eppendorf tubes (200mg in each) for further use.

\section{Deciphering Microbial Community Structures With Full-Length 16S /ITS rDNA TA-cloning}

The whole experimental process can be seen in our lab's publication [6]. For ITS amplification, primer ITS1 (5'-TCCGTA GGT GAA CCT GCG G-3') and ITS4 (5'-TCC TCC GCT TAT TGA TAT GC-3') were used. The PCR cycle is the same as in the report except that the extension time is $72^{\circ} \mathrm{C}$ for $60 \mathrm{~s}$.

\section{Sequence Edition, Taxonomy Classification and Statistical Analysis}

Positive colonies were subjected to Sanger sequencing using the same PCR primers. Each pair of bidirectional sequences was assembled as one single sequence with correct direction. Short sequences without primers on both ends were removed from the data and the residual vector bases and primer bases on both ends were deleted (LaserGene, DNAStar). Then the sequences were subjected to chimera checking using UCHIME [7] in mothur software [8]. Chimeric sequences were removed. Finally, the remained sequence was used for further taxonomic analysis. Simpson, Shannon, chao, and richness index as measures of alpha-diversity were calculated in Mothur [8].

\section{Phylogenetic Analysis of Sequences}

According to the taxonomic information, high-quality sequences were selected to make phylogenetic trees together with our own 16S rRNA gene sequences and ITS sequences respectively using software mega 6.0. Multiple alignments were generated with the ClustalW and the evolutionary distances were calculated using the Neighbour-Joining method. 


\section{Nucleotide Sequence Accession Numbers}

The refined sequences were deposited in the GenBank under the accession numbers KX603403-KX603652 (bacterial 16S rRNA) and KX911990-KX912161 (fungal ITS).

\section{Results and Discussion}

\section{Diversity analysis of GujingTribute Daqu microbial community}

Table1 provided the general information on microbial community diversity of Daqu samples. Using a 97\% similarity cutoff, 100 among 101 sequenced clones in H_Daqu showed species-level information, 115 among 148 sequenced clones in MH_Daqu provided species-level information. For Fungi, all 172 curated sequences of ITS clones can give species-level annotation. Chao is non-parametric species richness estimator calculated for each set of nsequences (nseqs, the number of successfully sequenced colonies). The chao value of MH_Daqu suggested there are about three hundred different bacterial species in the medium-high temperature Daqu of GujingTribute liquor. If $\mathrm{MH}-\mathrm{Daqu}$ has around 300 different species and every species can be observed reliably for 10-15 colonies in the TA-cloning experiment, approximately 5000 TA-cloning colonies have to be screened and bi-directionally sequenced. So it is not strange that the coverage is only about $79 \%$ after 160 colonies (with the nseqs as 148) were randomly picked to decode the MH-Daqu microbial community. However, the data in this report can still provide a general glimps on the microbial community in MH-Daqu, especially those most dominant species. Shannon and Simpson indices clearly indicated the relative diversity between two Daqu samples.

Table 1. Diversity analysis of GujingTributr Daqu samples

\begin{tabular}{llllllll}
\hline & Daqu & nseqs* & coverage & OTU & simpson & shannon & chao \\
\hline Bacteria & H_Daqu & 101 & 0.93 & 10 & 0.41 & 1.10 & 20.5. \\
& MH_Daqu & 148 & 0.79 & 41 & 0.15 & 2.63 & 273.50 \\
Fungi & H_Daqu & 90 & 0.97 & 6 & 0.45 & 0.96 & 6.50 \\
& MH_Daqu & 82 & 0.97 & 9 & 0.35 & 1.41 & 9.25 \\
\hline \multicolumn{7}{r}{$*$ nseqs: the number of successfully sequenced colonies. }
\end{tabular}

\section{Pantoea Species in GujingTribute Daqu}

Two bacterial species are unique as most dominant ones in GujingTribute Daqu and they are Pantoea agglomerans and Pantoea vagans (Fig.1, Table 2). While there are few reports on potential roles of Pantoea vagans in liquor fermentation, Pantoea agglomerans possesses several lines of evidence that it may play multiple roles during the fermentation process of liquor production. First, it has an ability to produce hydrogen [9] and thus may be acquired as a form of energy for some anaerobic bacteria. Second, it may play some roles as a valuable bioremediator to maintain the pit mud quality by preventing the penetration of some industrial contaminants into deeper parts of soil by biofilm formation [9]. Corsini et al (2016) [10] reported the presence of cop genes in the genome of Pantoea agglomerans related with copper homeostasis and potential for use as bioremediation agent. Son et al (2006) [11] found Pantoea agglomerans was a salt- and $\mathrm{pH}$-tolerant, insoluble inorganic phosphate-solubilizing bacterium that can efficiently work as an environment-friendly biofertilizer solubilizing insoluble phosphates. Third, it may have important antagonist roles in the early stage of the liquor fermentation process to balance between moulds growth and moulds-originated spoilage [12-13]. It can produce a number of antibiotics (phenazine, herbicolin, microcin, agglomerins, pantocins, andrimid, etc) [9] which could be used 
for combating some microbes during fermentation. Moreover, Pantoea agglomerans may contribute to the liquor's health-benefiting value by producing the low-molecular-mass lipopolysaccharide that has been revealing an extremely wide spectrum of healing properties in the prevention and treatment of a broad range of human and animal disorders, such as tumors, diabetes, various infectious diseases, hyperlipidaemia, allergy and stress-induced immunosuppression [14].
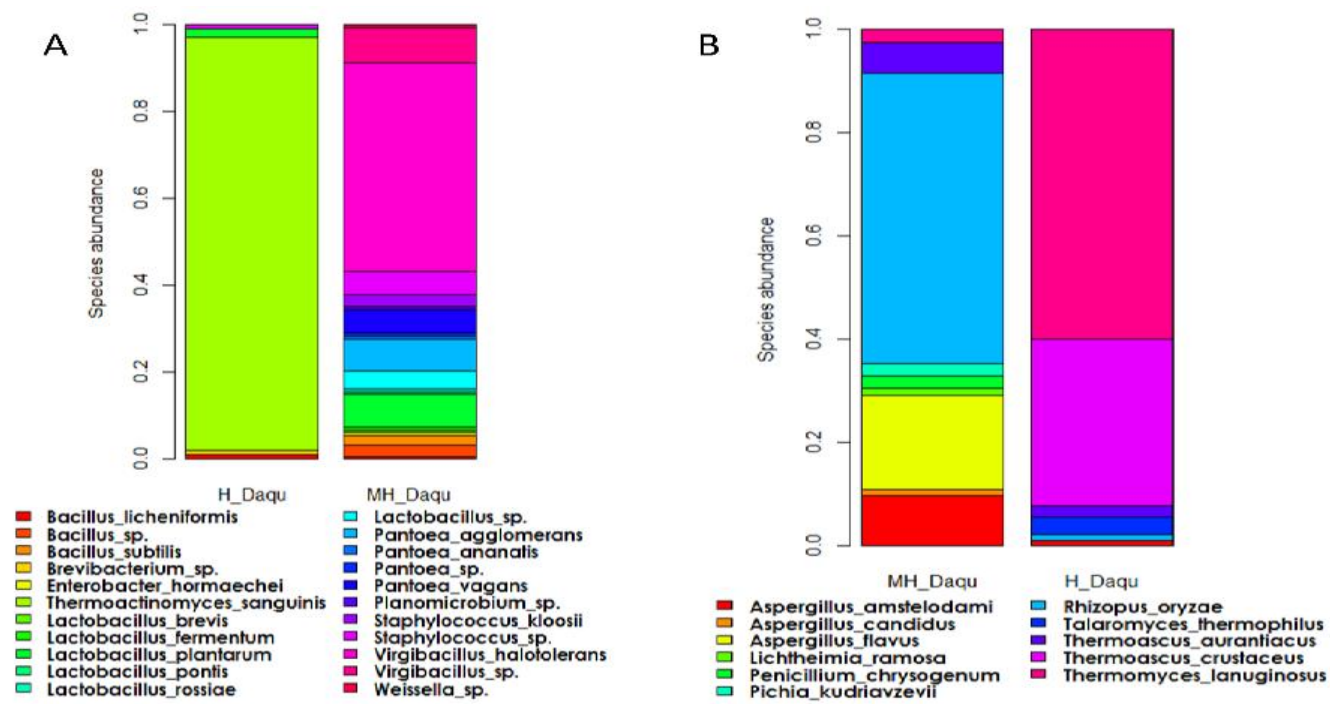

Figure 1. Prokaryotic (A) and eukaryotic (B) microbial compositions of Daqu samples at the species level.

Table 2. Most abundant five microbial species in Daqu of several famous brands of Chinese liquor

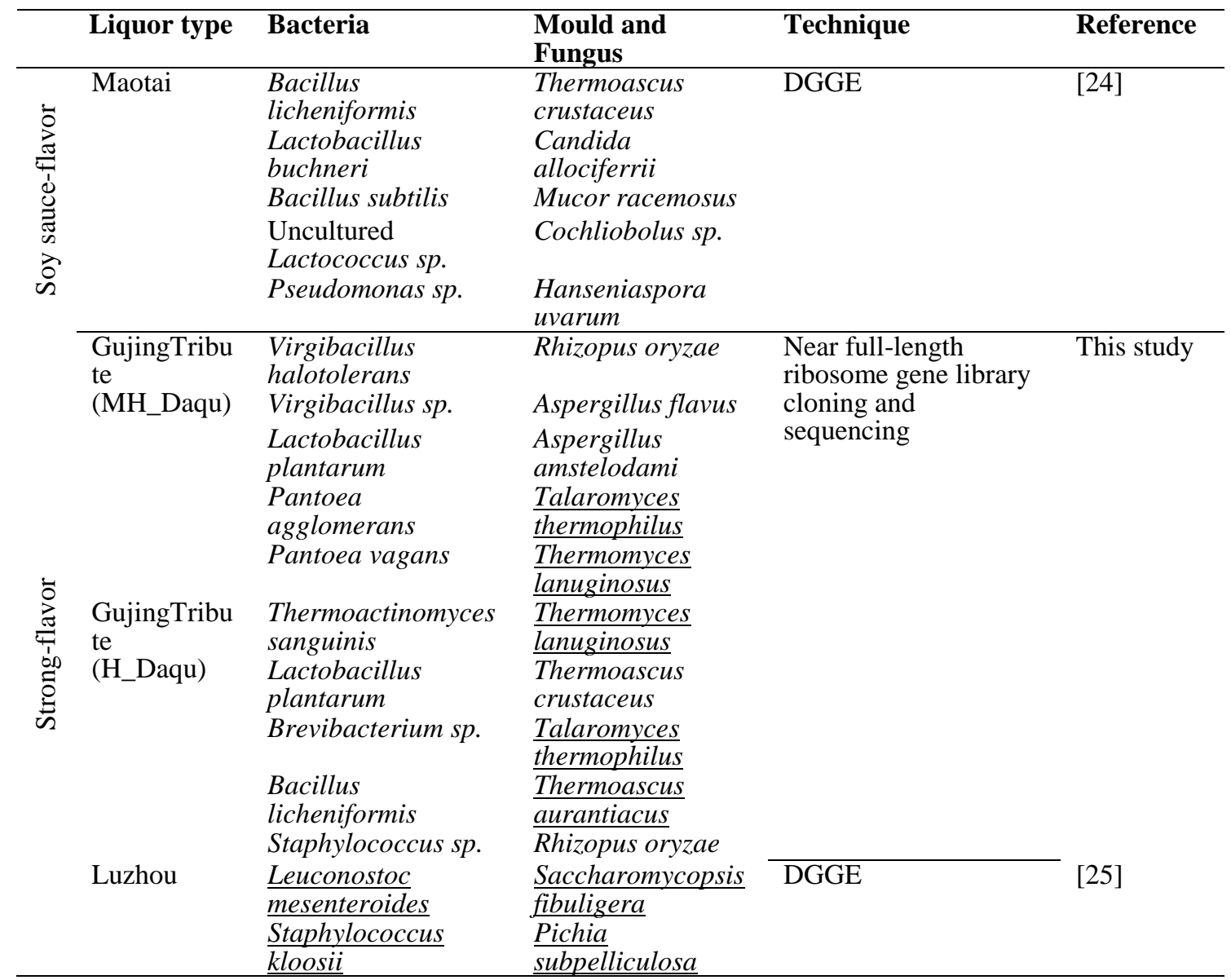


Table 2, cont. Most abundant five microbial species in Daqu of several famous brands of Chinese liquor

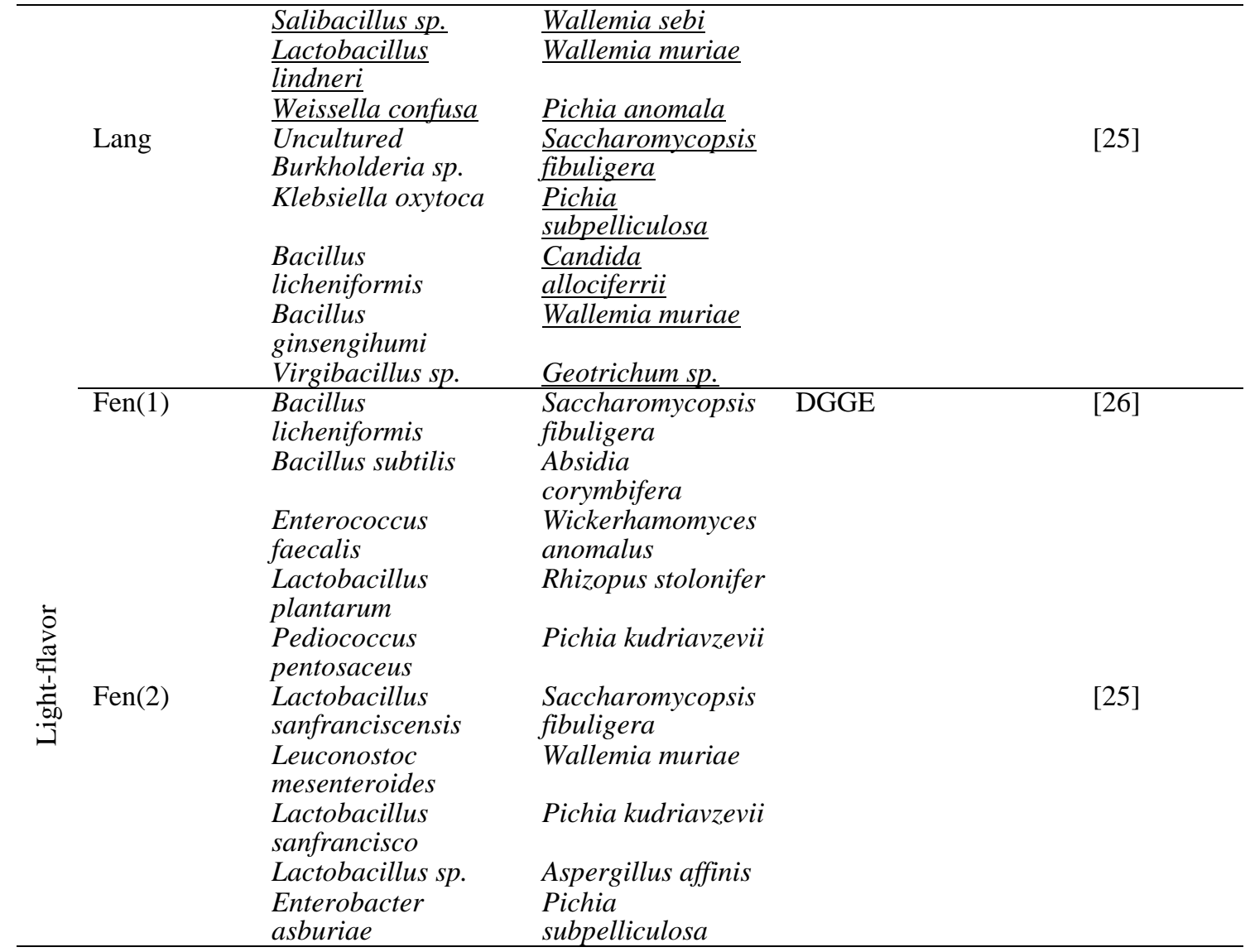

\section{Relatively Unique Dominant Species in GujingTribite Daqu}

Besides, in Table 2, Thermomyces only occurred in GujingTribute Daqu, not in any other types of Daqu. Indeed, there are 3 distinct species relatively unique as most dominant ones in Gujing Tribute Daqu: Talaromyces thermophiles, Thermomyces lanuginosus, and Thermoascus aurantiacus. There are few studies performed on Talaromyces thermophiles worldwide, but a plenty of studies have been reported for Thermomyces lanuginosus, and Thermoascus aurantiacus. The latter two species both can produce a series of useful enzymes for fermentation purposes. Aziz et al (2015)[15] investigated a lipase from Thermomyces lanuginosus in terms of their efficiency for the hydrolysis of safflower oil for the liberation of free linoleic acid used as a flavor precursor. Omar et al (2016)[16] employed immobilized lipase from Thermomyces lanuginosus to hydrolyze anhydrous milk fat and anhydrous buffalo milk fat, then used solid-phase micro-extraction gas chromatography/mass spectrometry to identify volatile flavouring compounds, and observed high amount of butanoic and hexanoic acids and other flavor compounds. Another report [17] suggested that Thermomyces lanuginosus lipase's one specific region can adopt a structural change at the water-lipid interface (which occurs during solid fermentation process of GujingTribute liquor), thus adjusting the levels of its lipase and esterase activities. Moreover, Pang et al (2016) [18] investigated butanol production employing fed-batch fermentation by Clostridium acetobutylicum using sugarcane bagasse hydrolysed by enzymes from Thermoascus aurantiacus. McClendon et al (2012) [19] compared Thielavia terrestris and Thermoascus aurantiacus for their ability to secrete enzymes that deconstruct biomass at high temperatures. Their conclusion is that T. aurantiacus produces a complement of 
secreted proteins capable of higher levels of saccharification, thus providing an excellent platform to develop a thermophilic system for the conversion of biomass to small molecules. All the above reported biological process of Thermomyces lanuginosus and Thermoascus aurantiacus may all be involved in GujingTribute liquor production.

\section{Relationship Between Daqu and Liquor Aroma Molecules'Origin}

Few studies have addressed the question to what extent the different types of liquor aroma come from Daqu. For Maotai liquor production, the ratio between high-temperature Daqu and cereals is about 1:1, which means Daqu itself takes a lot of proportion of fermentation stuff. For GujingTribute liquor production, Daqu is a mixture of H_Daqu and HM_Daqu at a ratio of 1:4, and the mixed Daqu is then mixed further with cereals at a ratio about 2.2:7.8. Every starting $100 \mathrm{~kg}$ cereals normally make $30-40 \mathrm{~kg}$ liquor. So theoretically, some aroma molecules maybe already enough in the Daqu while other aroma molecules are newly produced during the following fermentation steps.

The information of aroma mass transfer between Daqu and liquor is instructional for manipulation on aroma-producing strains in Daqu. For example, Bacillus licheniformis is one of the tetramethylpyrazine (TTMP) -producing microorganisms [20], and TTMP is a both aroma and health-benefiting chemical [21-22], so it is expected that GujingTribute liquor has a higher concentration of it by increasing the amount of TTMP-producing strains. The MH_Daqu of GujingTribute does contain Bacillus licheniformis, but this strain only occupies $0.68 \%$ in all MH_Daqu bacteria, while in Maotai Daqu Bacillus licheniformis is the most dominant species. This may explains why Maotai has a higher concentration of TTMP than other types of liquor [22-23].

\section{Conclusions}

To conclude, the main data presented in the paper are taxonomical, which illustrated the microbial compositions of the GujingTribute Daqu samples. Based on this, microbial population structures, especially the most abundant five species in Daqu of several types of classical Chinese liquor, were preliminarily compared, and it is clear that the combination patterns of five most abundant species in those Daqu samples were all different from each other based on known data. Note, if only surveying the most abundant five species, each of the seven different Daqu samples in Table 2 has one or more unique species absent in other six types of Daqu, which highlights a potential microbial basis for the production of unique set of aroma molecules for each type of liquor, though the most dominant species are mainly the ones degrading the feedstock during the fermentation process. Especially, there are 5 distinct species relatively unique as most dominant ones in GujingTribute Daqu (Table 2): Pantoea agglomerans, Pantoea vagans, Talaromyces thermophiles, Thermomyces lanuginosus, and Thermoascus aurantiacus. Characterization of Daqu microbial species, together with other microbial community information in the Zaopei and pit mud, provided a start point to decode the complicated relationship between microbial species and flavor substances in the GujingTribute liquor in the future.

\section{Acknowledgement}

This study was supported by NSFC (No.31071170), GujingTribute fund (2016-1), GREDBIO (201401) and HIT fund (hitwh200904, 2016GSF115022). 


\section{References}

[1] Gou, M. et al. Characterization of the microbial community in three types of fermentation starters used for Chinese liquor production. J. Inst. Brew. 121(2015)620-627.

[2] Li, H.H et al. Research on the volatile compounds in 2 kinds of Gujinggong liquor. J. Food Sci. Technol. 5(2015)1-10.

[3] Guo, W.J., Lu, J.C. Research on characteristic flavouring components of Gujinggong liquor. Liquor Mak. Sci. Technol.5(2001)83-85.(in Chinese)

[4] Huang, Y.M. et al. Analysis of flavoring substances in Gujing Gongjiu liquor by GC/MS. Liquor Mak. Sci. Technol. 7(2006)91-94. (in Chinese)

[5] Zhou, Q.W. et al. Research on volatile flavor components in Gujing Gongjiu liquor by two comprehensive dimensional gas chromatography-time of flight mass. Liquor Mak. 43(2) (2016) 75-81. (in Chinese)

[6] Zhang, H. et al. Employment of Near Full-Length Ribosome Gene TA-Cloning and Primer-Blast to Detect Multiple Species in a Natural Complex Microbial Community Using Species-Specific Primers Designed with Their Genome Sequences. Mol. Biotechnol. 58 (2016)729-737.

[7] Edgar, R.C. et al. UCHIME improves sensitivity and speed of chimera detection. Bioinformatics. 27(2011) 2194-2200.

[8] Schloss, P.D. et al. Introducing mothur: open-source, platform-independent, community -supported software for describing and comparing microbial communities. Appl. Environ. Micro. 75 (2009) 7537-7541.

[9] Zhu, D. et al. Fermentative hydrogen production by the new marine Pantoea agglomerans isolated from the mangrove sludge. Int. J. Hydrogen Ener. 33 (2008) 6116-6123.

[10]Corsini, G. et al. Draft genome sequence of a copper-resistant marine bacterium, Pantoea agglomerans strain LMAE-2, a bacterial strain with potential use in bioremediation. Genome Announc. 4(3)(2016) e00525-16.

[11] Son, H.J. et al. Solubilization of insoluble inorganic phosphates by a novel salt-and pH-tolerant Pantoea agglomerans R-42 isolated from soybean rhizosphere. Bioresour. Technol. 97(2006) 204-210.

[12]Dutkiewicz, J. et al. Pantoea agglomerans: a mysterious bacterium of evil and good. Part IV. Beneficial effects. Ann. Agric. Environ. Med. 23(2016) 206-222.

[13]Town, J., Dumonceaux, T.J. High-quality draft genome sequences of Pantoea agglomerans isolates exhibiting antagonistic interactions with wheat seed-associated fungi. Genome Announc. 4(2016) e00511-00516.

[14]Nakata, K., Inagawa, H., Soma, G.I. Lipopolysaccharide IP-PA1 from Pantoea agglomerans prevents suppression of macrophage function in stress-induced diseases. Anticancer Res. 31(2011) 2437-2440.

[15] Aziz, M., Husson, F., Kermasha, S. Optimization of the hydrolysis of safflower oil for the production of linoleic acid, used as flavor precursor. Int. J. Food Sci. (2015)594238. 
[16] Omar, K.A. et al. Effects of microbial lipases on hydrolyzed milk fat at different time intervals in flavour development and oxidative stability. J. Food Sci. Technol. 53(2)(2016)1035-1046.

[17] Skjold-Jørgensen, J. et al. Lipases that activate at high solvent polarities. Biochemistry. 55(2015) 146-156.

[18]Pang, Z.W. et al. Butanol production employing fed-batch fermentation by Clostridium acetobutylicum GX01 using alkali-pretreated sugarcane bagasse hydrolysed by enzymes from Thermoascus aurantiacus QS 7-2-4. Bioresour. Technol. 212(2016) 82-91.

[19] McClendon, S.D. et al. Thermoascus aurantiacus is a promising source of enzymes for biomass deconstruction under thermophilic conditions. Biotechnol. Biofuels. $5(1)(2012) 54$.

[20]Zhang, R., Wu, Q., Xu, Y. Aroma characteristics of Moutai-flavour liquor produced with Bacillus licheniformis by solid-state fermentation. Lett. Appl. Microbiol. 57(2013) 11-18.

[21] Meng, W., Xiao, D., Wang, R. Enhanced production of tetramethylpyrazine in Bacillus licheniformis BL1 by bdhA disruption and 2, 3-butanediol supplementation. World J. Microb. Biotechnol. 32(2016) 1-7.

[22]Zhao, Y., Liu, Y., Chen, K. Mechanisms and Clinical Application of Tetramethylpyrazine (an Interesting Natural Compound Isolated from Ligusticum Wallichii): Current Status and Perspective. Oxid. Med. Cell Longev. (2016) 2124638 (9 pages).

[23]Meng, W., Wang, R., Xiao, D. Metabolic engineering of Bacillus subtilis to enhance the production of tetramethylpyrazine. Biotechnol. Lett 37(2015) 2475-2480.

[24] Xiu, L., Kunliang, G., Hongxun, Z. Determination of microbial diversity in Daqu, a fermentation starter culture of Maotai liquor, using nested PCR-denaturing gradient gel electrophoresis. World J. Microbiol. Biotechnol. 28(6)(2012)2375-2381.

[25]Zhang, L. et al. Characterisation of microbial communities in Chinese liquor fermentation starters Daqu using nested PCR-DGGE. World J. Microbiol. Biotechnol. 30 (2014) 3055-3063.

[26]Zheng, X.W. et al. Complex microbiota of a Chinese "Fen" liquor fermentation starter (Fen-Daqu), revealed by culture-dependent and culture-independent methods. Food Microbiol. 31(2012) 293-300. 